\title{
Radiofrequency Ablation for Liver Metastases after Transarterial Chemoembolization: A Systemic Analysis
}

\author{
Chuan Xu' ${ }^{1}$, Peng-Hua Lv ${ }^{1 *}$, Xin-En Huang'*, Shu-Xiang Wang ${ }^{1}$, Ling Sun ${ }^{1}$, \\ Fu-An Wang ${ }^{1}$
}

\begin{abstract}
Background: This systemic analysis was conducted to evaluate tumor recurrence rate and one-year survival rate for patients with liver metastases received radiofrequency ablation after transarterial chemoembolization and introduce a new method of radiofrequency ablation by puncture navigation technology for single liver metastases after transarterial chemoembolization. Materials and Methods: Clinical studies evaluating tumor recurrence rate and one-year survival rate. Appling the innova trackvision software to process one liver metastases received transarterial chemoembolization and using radiofrequency ablation by puncture navigation technology to treat the liver metastases. Results: 3 clinical studies which including 235 patients with liver metastases after transaeterial chemoembolization were considered eligible for inclusion. Systemic analysis suggested that tumor recurrence rate was $23 \%(54 / 235)$, one-year survival rate was $76 \%(178 / 235)$. The new procedure was performed successfully and the patient received a good prognosis. Conclusions: This systemic analysis suggests that radiofrequency ablation is a good method for liver metastases after transarterial chemoembolization and could receive a relatively good prognosis.
\end{abstract}

Keywords: Liver metastases-radiofrequency ablation-transarterial chemoembolization

Asian Pac J Cancer Prev, 16 (12), 5101-5106

\section{Introduction}

Liver metastases will develop at some point during the course of the disease in up to $50 \%$ of these patients with gastrointestinal tumors (Michael et al., 2014). Patients with liver metastases generally receive chemotherapy, local treatment or resection. Traditional chemotherapy has played a limited role in the management of these relatively resistant tumors leaving many physicians with a nihilistic attitude toward hepatic malignancies (Tacher et al., 2013). Liver resection still remains a curative method for patients with hepatocellular carcinoma (HCC) and isolated liver metastases (Zhi et al., 2012), however, only $10-20 \%$ of patients actually have a resectable disease, because of either extensive disease or patient co-morbidities, or both. Transarterial chemoembolization (TACE) is used frequently for the treatment of small signal-nodule HCC or other liver metastases when other curative treatment are not possible. In real clinical practice, repeated TACE procedures are often needed, since the best response cannot always be achieved after one session of TACE (Georgiades et al., 2012; Jonggi et al., 2014). Radiofrequency ablation (RFA) is emerging as an effective local treatment for HCC or other liver metastases smaller than $3 \mathrm{~cm}$ in diameter. Although intrahepatic recurrence rates of up to 60 percent have been reported. Survival of patients with HCC and other liver metastases less than $3 \mathrm{~cm}$ treated by RFA competes with that of surgical candidaties (Lupo et al., 2007; Germani et al., 2010; Lewandowski et al., 2011). Both methods are effective in treatment of HCC and other liver metastases. Some studies combined TACE with RFA to treat HCC or other liver metastases and received a good prognosis (Zhi et al., 2012; Yang et al., 2014). Because TACE is a safe and effective alternative to hepatic resection and FRA for single-nodule HCC or liver metastases of $3 \mathrm{~cm}$ or smaller without vascular invasion especially for patients who are vulnerable to adverse events of treatments (Tacher et al., 2013; Michael et al., 2014). In clinical, we are usually using RFA to treat HCC or other liver metastases by ultrasound and computed tomography (CT) guiding (Kim et al., 2013). But we first do RFA under fluoroscopy puncture navigation technology (PNT) with the development of technology and introduce the process of this new treatment. According to this background, we hypothesize that RFA under PNT for Liver Metastases after Transarterial Chemoembolization could be established as an alternative treatment method.

1Department of Interventional Radiology, Subei People's Hospital of Jiangsu Province, Clinical Hospital of Yangzhou University, Yangzhou, 2Department of Chemotherapy, Jiangsu Cancer Hospital, Nanjing Medical University, Nanjing, Jiangsu, China *For correspondence: why77sina.com; huangxinen06@163.com 


\section{Materials and Methods}

\section{Literature Search}

We searched PUBMED, by using the following search term: (liver metastases), (radiofrequency ablation) and (transarterial chemoembolization). All clinical studies evaluating tumor recurrence rate and 1,3-year survival rate published in English prior to April of 2015 were identified. If samples of two studies overlap, only the least one was included. Additional articles were obtained from references within the articles identified by the electronic search. We did not consider meeting abstracts or unpublished reports.

\section{Inclusion and exclusion criteria}

We reviewed abstracts of all citations and retrieved studies. The following criteria were used to include published studies: (1) Clinical studies, conbined with liver metastases, radiofrequency ablation and transarterial chemoembolization; (2) The study was performed in accordance with the Helsinki Declaration (1964, amended in 1975 and 1983) of the world Medical Association. Eligibility criteria included histologically or cytologically verified metastatic and/or locally advanced primary tumor, the presence of at least one bidimensionally measurable lesion, a performance status (WHO) 2, age 18 years. Studies were exclued if one of the following existed: (1) duplicate data; (2) no sufficient data were reported.

\section{Data collection and analysis}

Selection of trial and data extraction: The titles and abstracts of publications identified according to the above search strategy were assessed independently for inclusion by two authors, the full text was selected for further assessment of the abstract suggests relevance. Disagreement was resolved by discussion. Data was extracted by independent authors. The following recorded data were extracted: author, publication data, and country of the first or corresponding author, the number of patients.

\section{New clinical technology}

One patient with gastric cancer received surgery on 2014-05-08, postoperative pathology was infiltrating adenocarcinoma. On 2014-11-17, upper abdominal enhanced CT revealed intrahepatic has a low density and diagnosis of gastric liver metastasis (figure 1). Although the patient could choose hepatic resection and systemic chemotherapy, she don't agree with surgery and systemic chemotherapy when communicated with her. Radiofrequency ablation was also excluded because of the tumor with a diameter of $5.45 \mathrm{~cm}$. At last, TACE was chosen to treat the patient on 2014-11-20. The procedure was successfully performed. On 2014-12-22, we checked upper abdominal enhanced CT which showed liver metastases was diminished significantly and the diameter was from $5.45 \mathrm{~cm}$ before procedure to $2.34 \mathrm{~cm}$ after TACE (figure 2). In order to prevent tumor progression, we communicated with the patient suggested further therapy with RFA by this new method, told her the procedure steps and obtained informed consent. The patient received RFA by fluoroscopy (Machine Type: Innova 530, workstation:
AW4.6) puncture navigation technology (PNT) on 201412-23. The patient received followed-up on 2015-0126, upper abdominal enhanced CT revealed that liver metastases necrosis and some lipiodial deposits (figure 3). In this procedure, the RFA was performed by fluoroscopy PNT, which was a new method for RFA.

\section{Procedure progress}

The patient with supine position on digital subtraction angiography (DSA) bed, received local disinfection and sterile covering.

Firstly, the patient received three-dimensional (3D) scaning and 3D reconstruction for upper abdomen. Because the patient did TACE before RFA, and has lipiodial deposits in tumor, we could see lipiodial under fluoroscopy and reconstruct 3D image for the tumor (figure 4). At the same time, we also could get bone $3 \mathrm{D}$ image (figure 4).

Secondly, we used the Innova Trackvision software provided by GE company to process after 3D scanning complete. 3D images of tumor and bone can be obtained. We could get more intuitive image space. Tumor image was changing in $3 \mathrm{D}$ space when we rotated $\mathrm{C}$ arm of DSA. It was convenient for us to select the best puncture point from different angles.

Then, we combined tumor with bone 3D images before selecting the entry point as to avoid the ribs. When the target point (usually tumor center) and the entry point (between 8 and 9 rib) selected, there would appear a line on the screen immediately, which was puncture path. In the process, we rotated the $\mathrm{C}$ arm and chose different levels, observed whether entry point was high or low, and ruled out whether puncture path was kept away from the chest (figure 5, 6, 7). The distance of the entry point and target point was measured, which was also the depth of radiofrequency ablation needle puncture distance.

Surface anchor point: rotating the $\mathrm{C}$ arm and choosing the right angle, as the entry point and target point merged one point (figure 8). At this point we can mark on body surface by continuous fluoroscopy which is the puncture point of skin.

Radiofrequency Ablation: We used 21G guiding (Terumo, japan) needle to puncture the tumor firstly and adjusted the needle under PNT (figure 9 and 10), and then RFA needle (Medsphere, US) deepen into the tumor along with the guiding needle, expanding ablation needle (the length of the probe was $3 \mathrm{~cm}$ ) when arrived the tumor center. Checking again before ablation to confirm whether radiofrequency ablation needle in the lesion. Rotating the $\mathrm{C}$ arm (anteroposterior and lateral position, shown in figure 11 and 12), if the needle not cover in the tumor, we should adjust the needle until in the correct position. Our RFA needle was in the correct position, so we started ablation (ablation temperature was 100 degrees, last for 5 minutes) and ended the procedure successfully.

\section{Results}

There were 195 papers relevant to the search words by the end of April of 2015. Via steps of screening the title and reading the abstract, three studies were identified 
(Mark et al., 2002; Martin et al., 2009; Zhi et al., 2012). These studies had been carried out in China, USA, and Japan. The following outcomes were presented in at least all studies and extracted for combined analysis: tumor

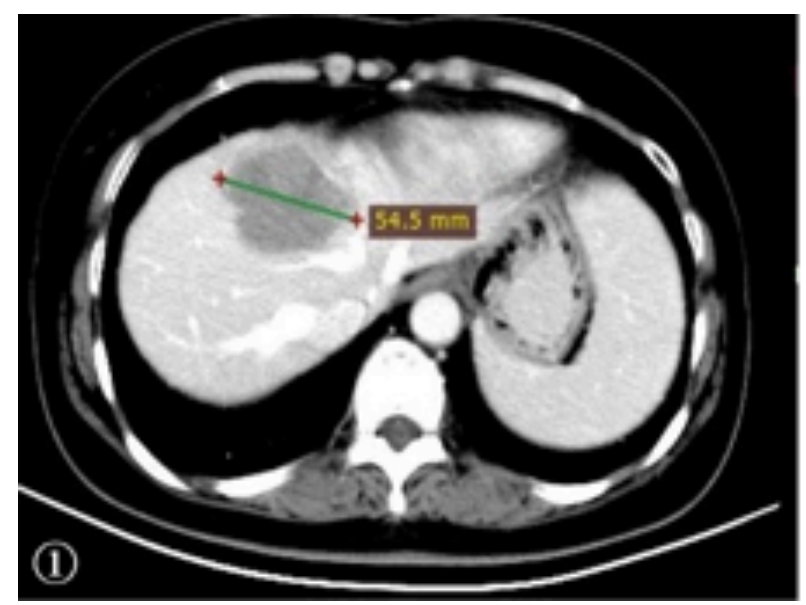

Figure 1. Upper Abdominal Enhanced CT (2014-1117): there is a Low Density in the Liver and Diagnosis of Gastric Liver Metastasis, the Diameter was $5.45 \mathrm{~cm}$.

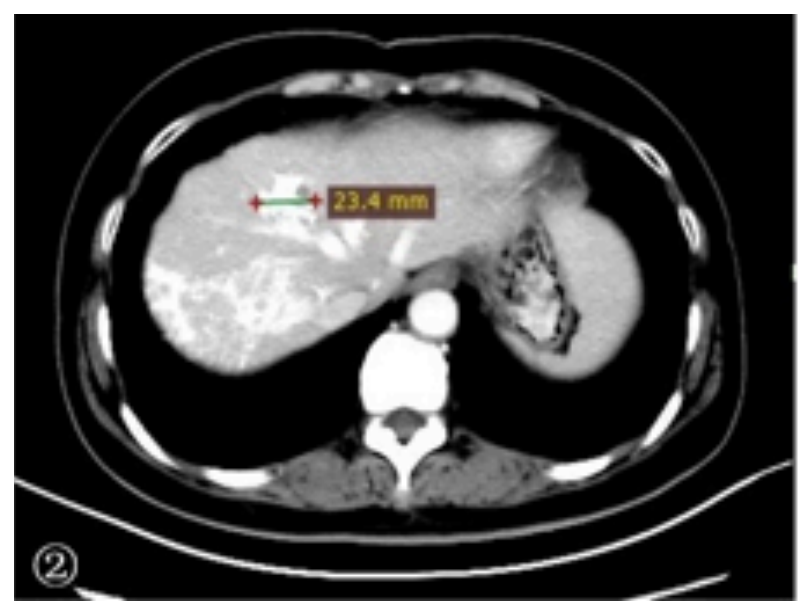

Figure 2. Upper AbdominalEnhanced CT (201412-22): Gastric Hepatic Metastasis was Diminished Significantly after TACE and the Tumor Diameter was $2.34 \mathrm{~cm}$

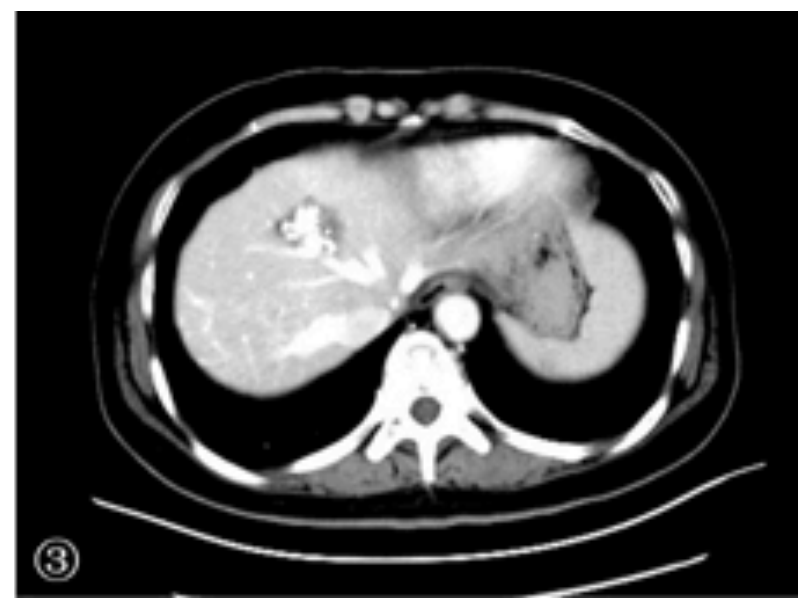

Figure 3. Upper Abdominal Enhanced CT (201501-26): There Some Necrosis Tissue around Gastric Hepatic Metastases after RFA and Some Lipiodial Deposit in Tumor recurrence rate and one-year survival rate. Characteristics of studies included in this analysis are presented as shortterm outcomes: tumor recurrence rate of Zhi et al. was $61 \%$, of Martin et al. was 69\%, of Mark et al. was 54\%. One-year survival rate of Zhi et al. was 100\%, of Martin et al. was $93 \%$, of Mark et al. was $87 \%$. Totally, 235 patients

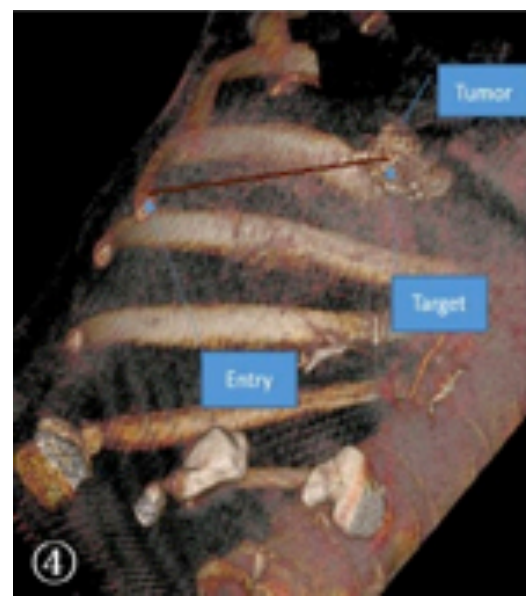

Figure 4. The Patient Received 3 D Scanning and 3 D Reconstruction for Upper Abdomen. We could reconstruct $3 \mathrm{D}$ image for tumor and bone

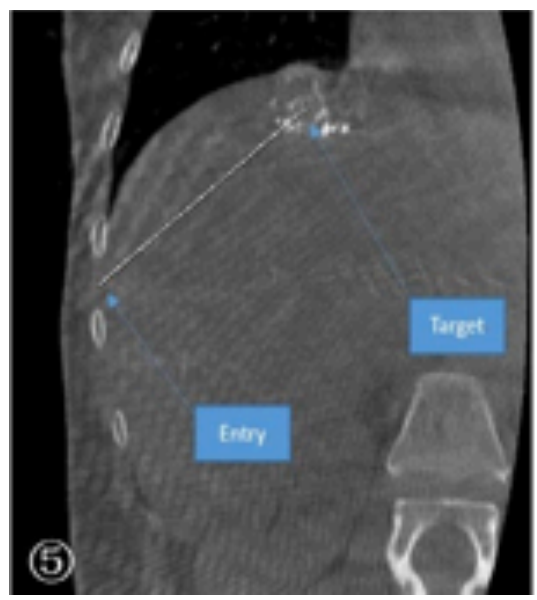

Figure 5. Coronal section: Puncture Path Can be Viewed from any Direction to See Weather Reasonable, to Avoid through the Chest and Prevent the Occurrence of Pneumothorax

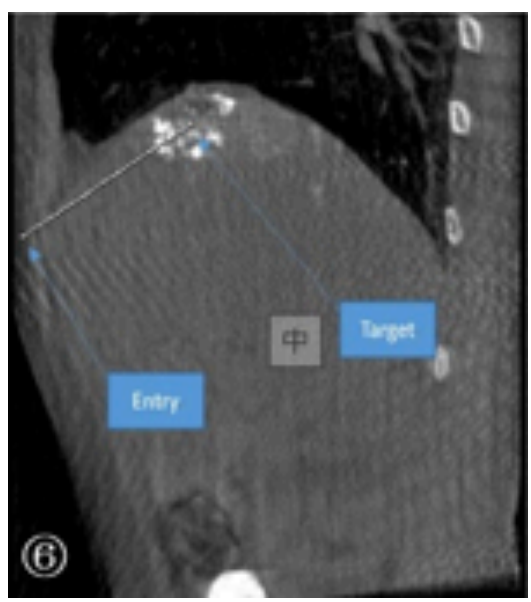

Figure 6. Median Sagittal Section: Puncture Path was Kept Away from the Chest 
Chuan Xu et al

were enrolled and 178 patients achieved good response. The patient with gastric liver metastases received the new technology and the procedure performed successfully.

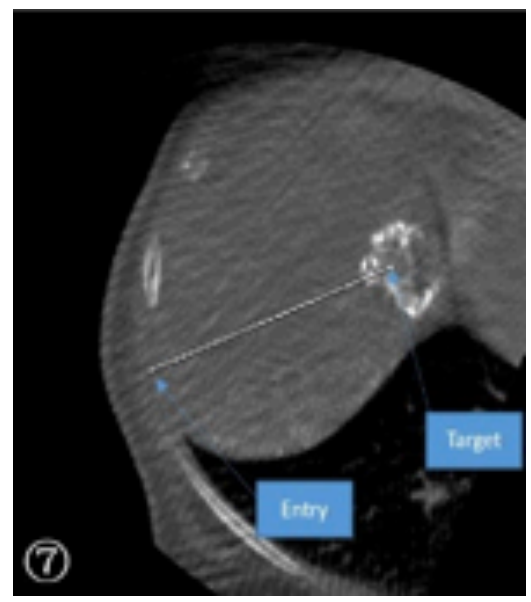

Figure 7. Transverse Section: Puncture Path was Kept away from the Chest

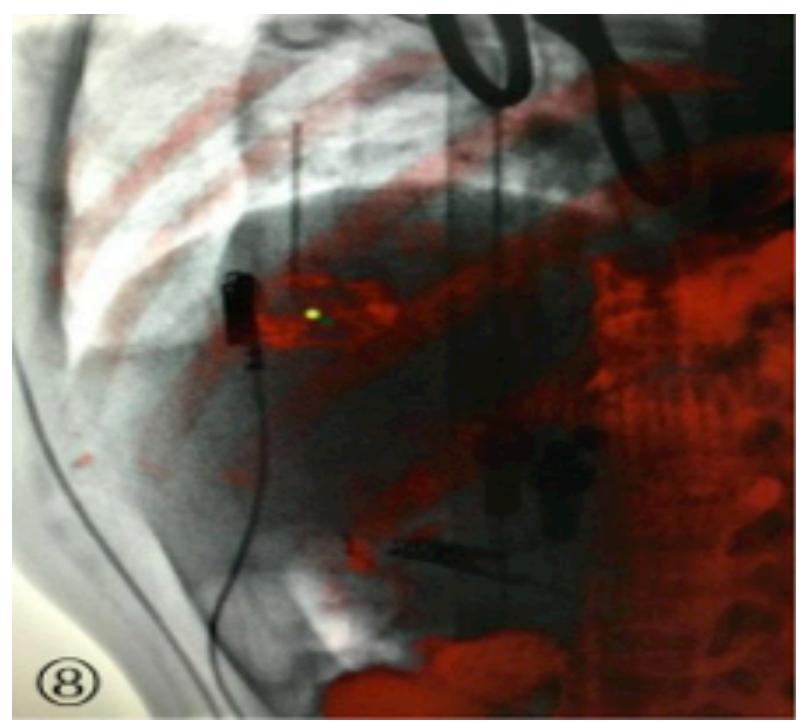

Figure 8. The Entry Point and Target Point Merged one Point, Which was the Puncture Point of Skin

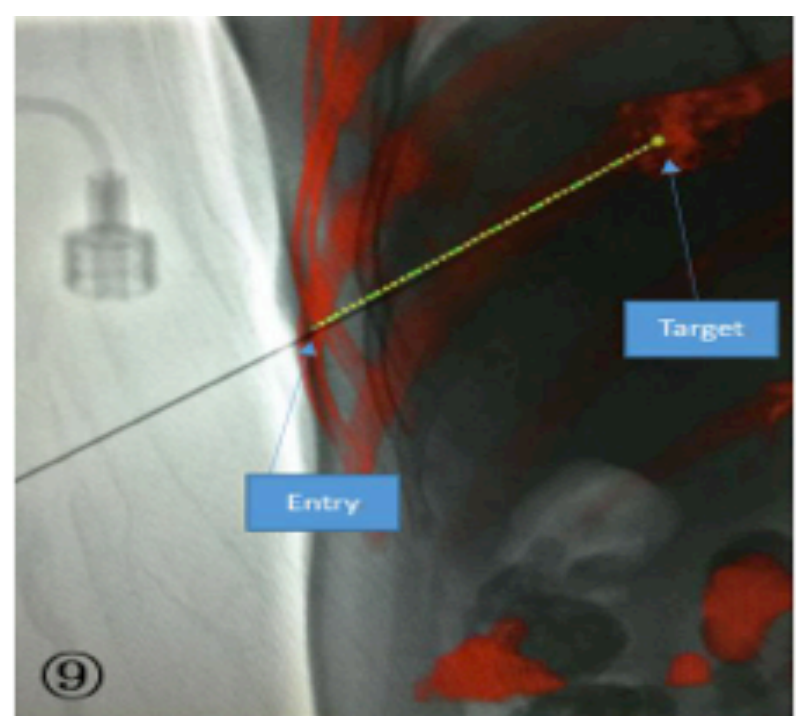

Figure 9. Puncturing the Tumor by 21G Guiding Needle Under PNT and the Path was Match to the Anteroposterior Positon

5104 Asian Pacific Journal of Cancer Prevention, Vol 16, 2015

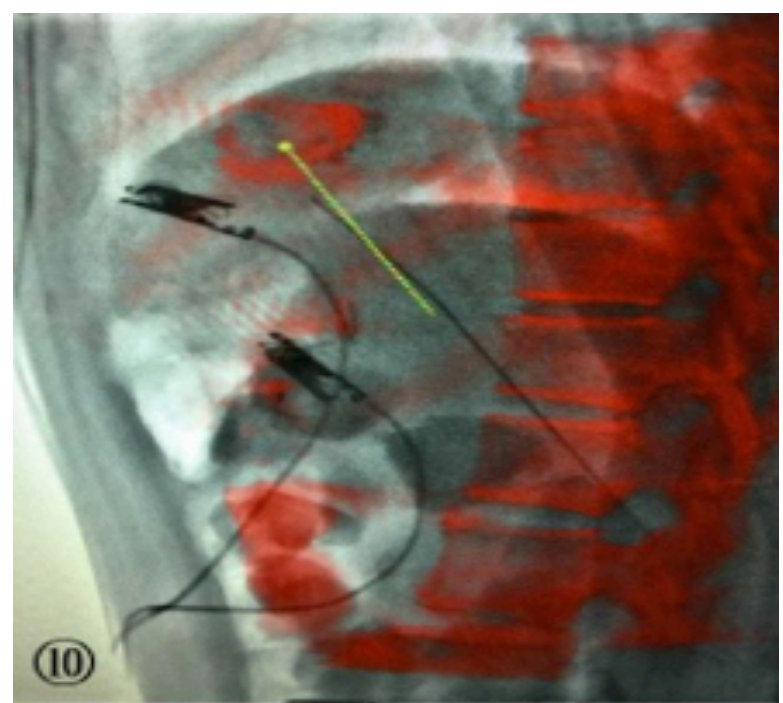

Figure 10. Puncturing the Tumor by 216 Guiding Needle Under PNT and the Path was Match to the Lateral Position

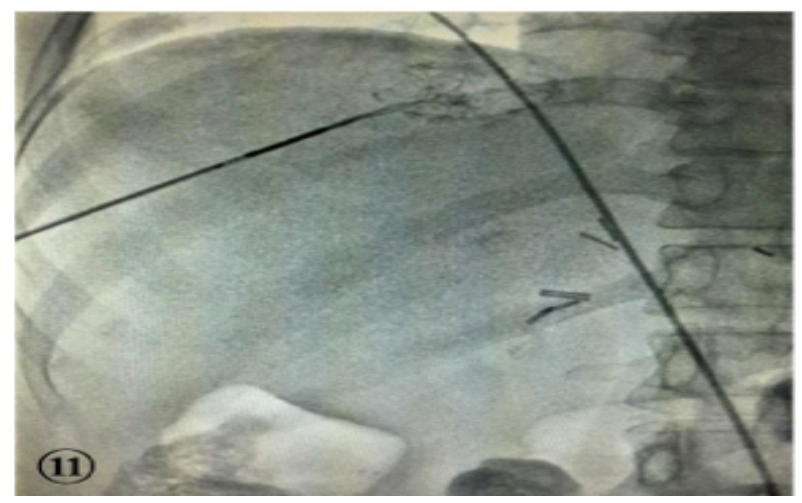

Figure 11. Our RFA Needle was in the Correct Position from Anteroposterior Positon

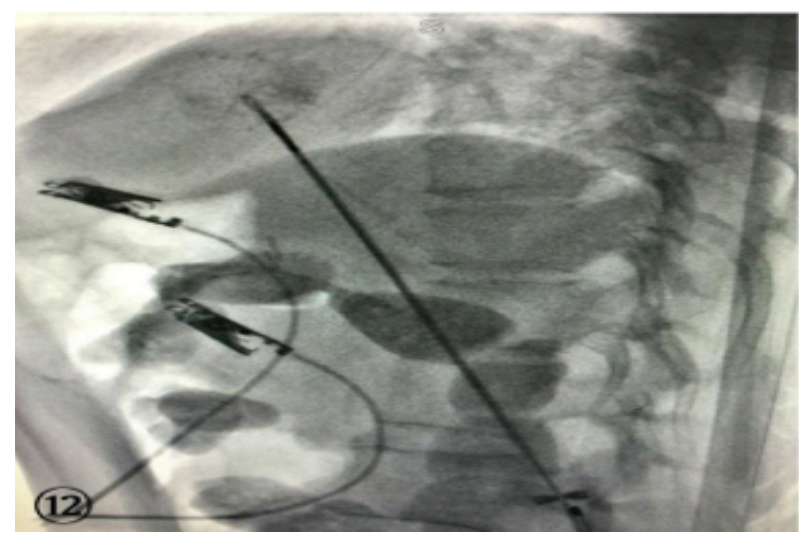

Figure 12. Our RFA Needle was in the Correct Position from Lateral Position

Upper abdominal enhanced CT on 2015-01-26 revealed that no new hepatic metastases, the lesion was good controlled, there was necrosis surrounding the tumor and reached expected effect.

\section{Discussion}

Nonoperative management of patients with advanced primary and metastatic hepatic malignancies has 
become a topic of much interest in recent years as new nonresectional techniques have been developed (Nakai et al., 2005). Athough each form of therapy has met with its share of enthusiasm and criticism very little has been published regarding combined nonresectional therapies. Of those who present with metastases confined to the liver, only $8-27 \%$ are eligible for surgical resection, whereas, the remaining majority of patients rarely survive 5 years (Bloomston et al., 2002). Kosuke shows that introhepatic recurrence rate was 54\% after RFA, there was no statistical difference compared with resection (Kosuke et al., 2013). A study shows that tumor size $3 \mathrm{~cm}$ were independent predictors of mortality. If tumor size less than $3 \mathrm{~cm}$, the type of treatment (RFA vs resection) did not affect survival (Vigano et al., 2008). Survival of patients with HCC and other liver metastases less than $3 \mathrm{~cm}$ treated by RFA competes with that of surgical candidaties.

Usually, we are performing radiofrequency ablation for patient with HCC or other liver metastases by CT or ultrasound, but we cannot obtain intuitive 3D image by those methods (Pellerin et al., 2013). We should take accurate positioning before puncture and adjust the position repeatedly when puncture into the center of tumor, it would inevitably cause liver injury. But this new method of treatment could make us to get intuitive 3D image before radiofrequency ablation, determine the entry and target point before procedure, choose the best puncture point and path, make the procedure more intuitive and convenient, avoid damaging the surrounding organs and the repeated puncture while adjusting the puncture direction by CT or ultrasound. But if patients with HCC or other liver metastases no received TACE before RFA, there were no lipiodial deposits in tumor, and the tumor cannot be tagged under fluoroscopy. We cannot reconstruct 3D image for the tumor and cannot conform the target point for patients need RFA, so the puncture navigation won't be performed. We can avoid damaging blood vessels by CT or ultrasound guiding for patient received RFA (Kang et al., 2002; Salem et al., 2013), the risk of this new method is higher if the tumor is near vessels because major blood vessels cannot be shown under fluoroscopy.

In conclusion, this systemic analysis suggests that RFA is a good method for liver metastases after TACE and could receive a relatively good prognosis. RFA under PNT is a novel treatment especially for patients with HCC or other liver metastases (less than $3 \mathrm{~cm}$ in diameter) received TACE, which provides a new treatment method and makes the procedure more intuitive and convenient. Future studies are needed to evaluate the efficacy of this procedure.

\section{Acknowledgements}

I would like to express my gratitude to all those who performed the procedure. A special acknowledgement should be shown to Professor Peng Hua Lv who helped work out an outline of this paper and useful instructions all through my writing. Finally I wish to extend my thanks to Fu An Wang and Wen Nuo Huang who supplied me with references of great value.

\section{References}

Bloomston M, Binitie O, Fraiji E, et al (2002). Transcatheter arterial chemoembolization with or without radiofrequency ablation in the management of patients with advanced hepatic malignancy. Am Surg, 68, 827-31.

Georgiades C, Geschwind JF, Harrison N, et al (2012). Lack of response after initial chemoembolization for hepatocellular carcinoma: dose it predict failure of subsequent treatment? Radiology, 265, 115-23.

Germani G, Pleguezuelo M, Gurusamy K, et al (2010). Clinical outcomes of radiofrequency ablation, percutaneous alcohol and acetic acid injection for hepatoceluller carcinoma: a meta-analysis. J Hepatol, 52, 380-88.

Jonggi C, Ju HS, Yong MS, et al (2014). Clinical significance of the best response during repeated transarterial chemoembolization in the treatment of hepatocellular cacinama. J Hepatol, 60, 1212-18.

Kang HK, Jeong YY, Choi JH, et al (2002). Three-dimensional multi-detector row CT portal venography in the evaluation of portosystemic collateral vessels in liver cirrhosis. Radio Graphics, 22, 1053-61.

Kim YS, Lim HK, Rhim H, et al (2013). Tenyear outcomes of percutaneous radiofrequency ablation as first-line therapy of early hepatocellular carcinoma: analysis of prognostic factors. J Hepatol, 58, 89-97.

Kosuke M, Toru B, Aikira C, et al (2013). Hepatic resection combined with radiofrequency ablation for initially unresectable colorectal liver metastases after effective chemotherapy is a safe procedure with a low incidence of recurrence. Am Surg, 83, 1527-31.

Lewandowski RJ, Geschwind JF, Liapi E, et al (2011). Transcatheter intraarterial therapies: rationale and overview. Radiology, 259, 641-57.

Lupo L, Panzera P, Giannelli G, et al (2007). Single hepatocellular carcinoma ranging from 3 to $5 \mathrm{~cm}$ : radiofrequency ablation or resection? HPB (Oxford), 9, 429-34.

Mark B, Odion B, Elie F, et al (2002). Transcatheter arterial chemoembolization with or without Radiofrequency Ablation in the Management of Patients with Advanced Hepatic Malignancy. Am Surg, 68, 827-31

Martin RC, Robbins K, Tomalty D, et al (2009). Transarterial chemoembolisation (TACE) using irinotecan-loaded beads for the treatment of unresectable metastases to the liver in patients with colorectal cancer: an interim report. World $J$ Surg Oncol, 7, 80-92.

Michael V, Ali H, Thomas JW, et al (2014). Unresectable solitary hepatocellular carcinoma not amenable to radiofrequency ablation: multicenter radiology-pathology correlation and survival of radiation segmentectomy. Hepatology, $\mathbf{6 0}$, 192-201.

Nakai M, Shiraki T, Higashi K, et al (2005). Low-output radiofrequency ablation combined with transcatheter arterial oilychemoembolization for hepatocellur carcinoma. Nihon Lgaku Hoshasen Gakkai Zasshi, 65, 124-26.

Pellerin O, Lin M, Bhagat N, et al (2013). Can C-arm conebeam CT detect a micro-embolic effect after TheraSphere radioembolization of neuroendocrine and carcinoid liver metastasis? Cancer Biother Radiopharm, 28, 459-65.

Salem R, Miller FH, Yaghmai V, et al (2013). Response assessment methodologies in hepatocellular carcinoma: complexities in the era of local and systemic treatments. $J$ Hepatol, 58, 1260-62.

Tacher V, Lin M, Chao M, et al (2013). Semiautomatic volumetric tumor segmentation for hepatocellular carcinoma: comparison between C-arm cone beam computed tomography and MRI. Acad Radiol, 20, 446-52. 


\section{Chuan Xu et al}

Vigano L, Ferrero A, Lo Tesoriere R, et al (2008). Liver surgery for colorectal metastases: results after 10 years of follow-up. Long-term survivors, late recurrences, and prognostic role of morbidity. Ann Surg Oncol, 15, 2458-64.

Yang HJ, Lee JH, Lee DH, et al (2014). Small single-nodule hepatocellular carcinoma: comparison of transarterial chemoembolization, radiofrequency ablation, and hepatic resection by using inverse probability weighting. Radiology, 271, 909-18.

Zhi VF, Francesco P, Laurence N, et al (2012). Combined Hepatic Arterial Embolization and Hepatic Ablation for Unresectable Colorectal Metastases to the liver. Am Surg, 78, 1243-48. 